\title{
Study on using design circuits to implement a Mobile Robot systems for Monitoring and Control
}

\author{
Changgeng $\mathrm{Yu}^{1, \mathrm{a}}$, Xiangxu Xie ${ }^{1, \mathrm{~b},{ }^{*}}$, Fulian $\mathrm{Li}^{1, \mathrm{a}}$ \\ ${ }^{1}$ School of Mechanical and Electronic Engineering, Hezhou University, No.18, xihuan Road, \\ Hezhou,China \\ ayuchanggen66@163.com, bthxiexx@163.com
}

Keywords: Mobile robot; Arduino platform; Video monitoring; Wireless transmission

\begin{abstract}
The mobile robot system can actually be applied to some of the places reconnaissance and monitoring, and the use of wireless networks, making the operating system more flexible, so that movement of the machine by monitoring and investigation without the shackles of cable. In this paper, we present the design and implementation of a mobile system with wireless for remote monitoring and control. It was composed of a multiple sensors, a control board, a camera video capture and a wireless communication module. The robot prototype was manufactured and experimented. Experiment results of typical cases verified its flexibility and reliability.
\end{abstract}

\section{Introduction}

With the progress of science and technology, mobile robot for monitoring are palying more and more important roles[1]. A wide range of automation, control equipment and other equipment for centralized interconnected has become a trend in many field, such as factory automation, machinery, and manufacturing, if use of Internet technology and processing equipment, robotics, control systems, and on-site linking, you can achieve Internet environment automation[2]. Undoubtedly, the robot control and network technology will have a very important theoretical significance and wide application prospect. Classical control theory, we know that the basic control system typically comprises modules, such as information detection module, the controller module, and actuator module. These key module state is directly related to the effectiveness and stability control systems. With the increasing level of multi-functional smart device technology, more and more enterprises, the company began research and design of intelligent robot platform, with further research, greatly accelerated the pioneering research in the field of multi-directional intelligent robot devices.

Self-localization is a basia issue in the mobile robots[3-7]. As the diversity of monitoring mobile robot module. By installing different hardware sub-module, one can save costs, on the other hand can make the model of refining. In order to improve the degree of artificial intelligence of the mobile robot, and can respond quickly in the implementation process given the task order, then the mobile robot has a necessary prerequisite is to have the ability to carry different types of sensor functions. Mobile robots get information from the sensor, and its data is processed in the microcontroller through the relevant algorithm after obtaining the policy, then the appropriate action to achieve through the implementation of the module [8].

Through the modular structure of the hardware was designed, according to the actual situation of need, ready to install sensors to achieve the different functions and monitoring mobile robot has wireless network communication capabilities. In order to monitor real-time health of the mobile robot, and the need to move the robot status information and run dynamic information, including road run dynamic status, motor information, servo information, speed information, etc. sent to the host computer, in order to obtain these useful Real-time monitoring of running status information to the mobile robot, while the host computer can control the operation of the mobile robot, such as acceleration, change of direction, set various parameters related to the mobile robot to achieve manual control. A good, convenient, and user-friendly surveillance mobile robot allows us to gather critical data in a timely manner, and the data necessary processing. 


\section{System Overview}

This system mainly consists of two parts: the lower part of the mobile robot machine and PC main control section. The next bit machine mobile robot part, mainly consists of two parts: the network portion and the camera portion Arduino control board, the webcam is mainly used for video information collection, Arduino control board is mainly used for steering gear, DC motor control and sensor control, movement control and sensor information collection car. Master PC-side main control software system, the next crew to operate the mobile robot control, at the same time, be able to obtain information on the webcam video, webcam can be controlled, and the camera capture the information processing software. Relationship links the system shown in Fig 1.The design of robot can be divided into five steps:(1) According to the design requirements, determine the control program. (2) the use of Altium Designer designed hardware schematic diagrams. (3) Draw program flow chart, using the $\mathrm{C}$ programming language, Arduino simulation debugging. (4) Each element is soldered on the PCB, and is programmed into the microcontroller. (5) In order to debug control functions.

\section{Hardware Design}

Mobile surveillance robot consists of Arduino UNO R3 MPU circuit, Robot-Link V4.0 AR WIFI module, L298N drive module, motor, car chassis, power supply modules and other components [9]. The operating structure of the graph is shown in Fig 2.

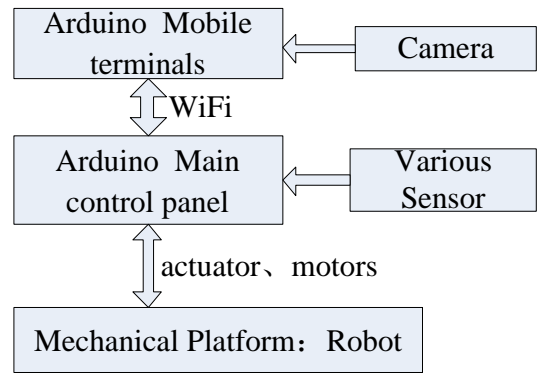

Figure 1. System link

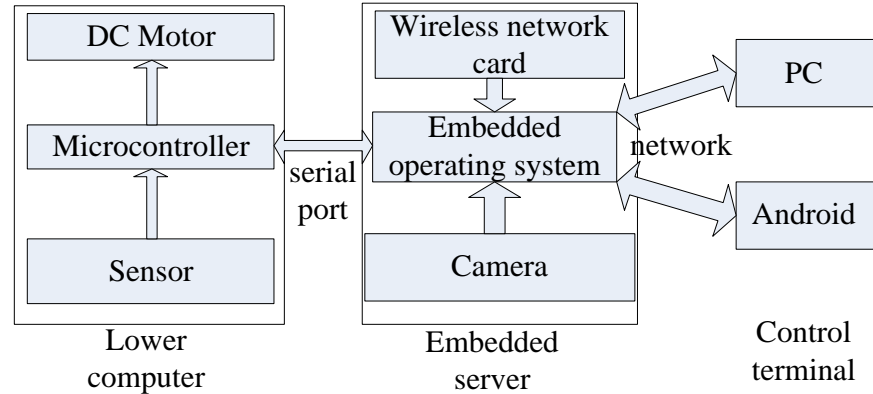

Figure 2. control system structure

(1) Arduino MPU Circuit. The main control circuit board: Arduino UNO R3 MPUs ATMEGA328P chip as a control unit. Arduino is a USB interface based on open source Simple I / O interface board (including 12-channel digital GPIO, 4-channel PWM output, channel 10bit ADC input channel 6-8), and has a similar use Java, C language IDE integrated hardware and software. Arduino UNO is the latest version of the Arduino USB interfaces series. Fig 3 is a development board Arduino UNO and parameter descriptions.

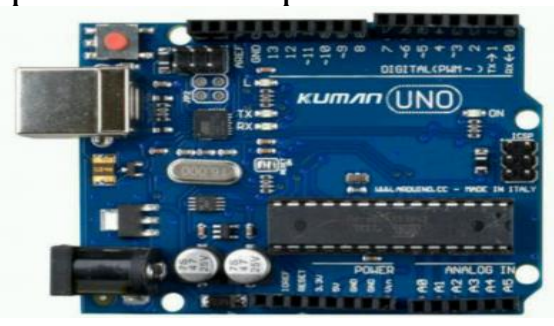

\begin{tabular}{|l|c|}
\hline \multicolumn{1}{|c|}{ Resource } & Explain \\
\hline Microcontroller Models & ATmege328 \\
\hline EEPROM & $1 \mathrm{~KB}$ \\
\hline SRAM & $2 \mathrm{~K}$ \\
\hline Reservoir & $32 \mathrm{~K}$ \\
\hline DC Apply & $5 \mathrm{~V}$ \\
\hline Analog Input Foot & 6 \\
\hline DigitalLO Foot & 14 \\
\hline TCK & $16 \mathrm{MHz}$ \\
\hline Serial Port & 1 \\
\hline
\end{tabular}

Figure 3. Arduino UNO parameters

(2) Wireless Wifi Routing Module. Wireless WiFi routing module used in processing chip is MT7620N, this router is Open WRT system, Open WRT is a highly modular, highly automated embedded Linux systems, components, and has a strong network scalability is often used industrial equipment, telephone, small robots, intelligent home, routers and VOIP devices. The routing module can be used as forwarding station to achieve load USB camera and serial communication with the microcontroller. 
(3) Video Capture System. Video capture system by installing cameras at the remote scene of the completion of the real-time monitoring, and make the appropriate controls based on the collected information and feedback, when the abnormal situation occurs, it is possible rapid response, positive response to help the operator to provide the right decisions. Video surveillance technology based on embedded technology have many advantages, such as small size, high stability, disorderly site duty, real good and simple structure, the video surveillance system consists of Video Capture System, PTZ camera control system, A signal transmission system and a video processing system.

(4) The Motor Drive Module. Multifunctional motor-driven drive module: using as the L298N motor driver chip. L298N has a high voltage, high current, high frequency response of the full-bridge driver chip, one can control four L298N DC motor, and with a control enable. The motor driver chip drives capability, easy operation, good stability, excellent performance. L298N enable terminal can add level control; you can also take advantage of MCU software control to meet the needs of complex circuits. L298N is an internal H-bridge with two high voltage and high current full-bridge driver chip that can be used to drive DC motors, stepper motors. In addition, L298N drive power is larger, according to the input voltage and the output of different voltage and power to solve the problem of insufficient load capacity [10].

(5) Power Module. Power module: from 2200mAh 7.4 8A Lithium battery protection board composition. By 7805 chip regulator by $0.1 \mathrm{uF}$ and $470 \mu \mathrm{F}$ capacitor filter.

\section{Software Design}

PC control program uses C\# programming language, can be realized by Robot-Link V4.0 AR WIFI module sends a command to the next crew and reception. But also through Wireless Module PTZ image signal transmission to the host computer image display window.

Lower machine Arduino control board uses the Arduino IDE development. Arduino language is based on wiring language development, is avr-gcc library of the second package, the microcontroller does not require much basic programming foundation. Because all the advantages of Arduino, a growing number of professional hardware developers have started using Arduino or to develop their projects, products; more and more software developers to use Arduino to enter the field of hardware development, networking and so on.

PC clients running control terminal control program must first establish a connection request to wait for the server-side response, the connection is successful you need to complete the function shown in Fig. 4.

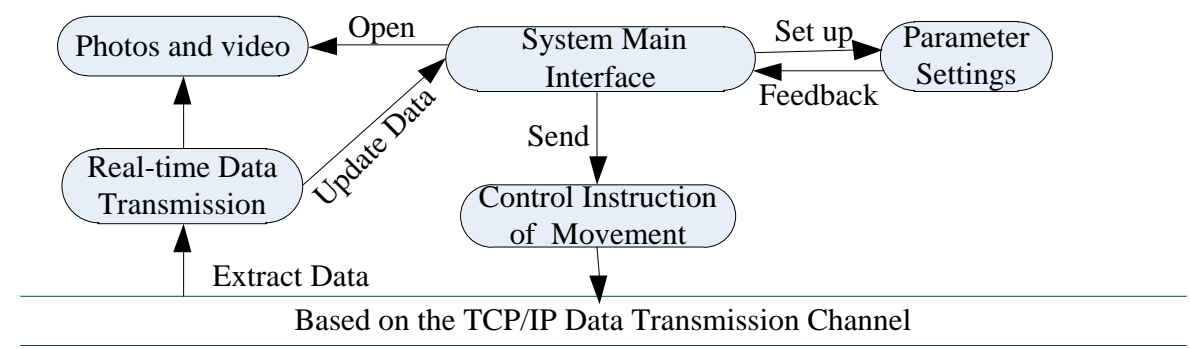

Figure 4. PC control terminal overall function block diagram

PC Remote Control client software overall function, contains three main parts: The video image decoding display, data processing and display, and transmit control information data.

Under-Bit machine is the executive body of the robot, the direct control of various external devices and sensors, mainly responsible for receiving serial commands, parse serial command, the drive hardware work. At the same time, sensor data collection, according to the agreement package, sent from the serial port to the server, which runs on the microcontroller, the main program flow shown in Fig 5. Under normal operating conditions, the procedure loops, receiving commands, parse the command, execute the command, returns the result, according to the parameters set PWM control commands to control DC motor speed to control the speed and direction of movement of the robot. 


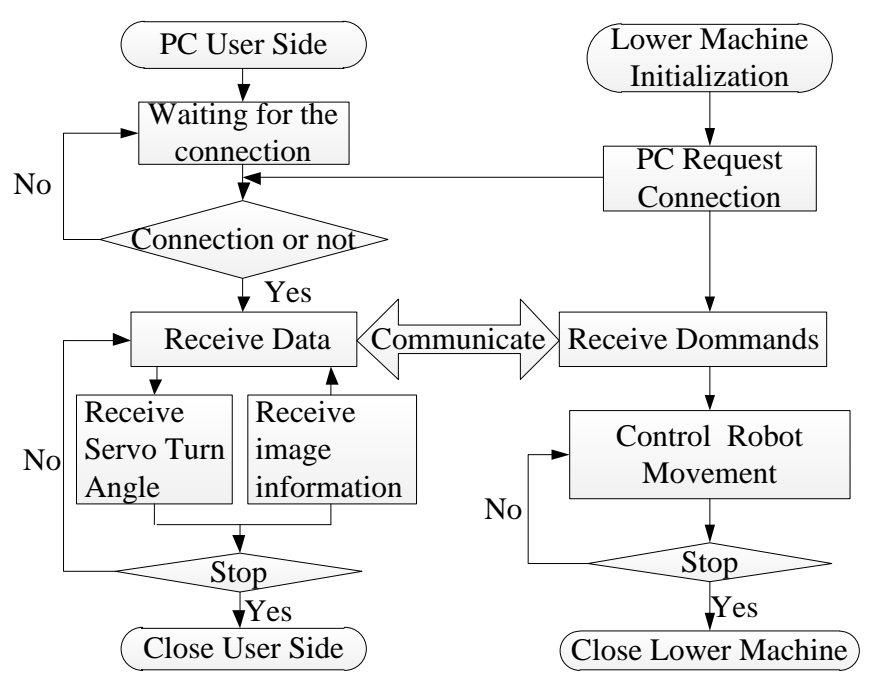

Figure 5: Lower computer program execution flow

\section{Prototype and Experiments}

After complete the system design, it is necessary to repeatedly test various parts of the found a potential problem and provide the basis for improving design, system testing is an important step design meets the requirements of the verification. System testing required by the functions and modules, the first test of the hardware system, ensure a stable operating environment, followed by the test system software to ensure the normal start the server application, and then test the control terminal, ensure the normal response to user actions, send the right control command and parsing the video data, and finally test the real-time communication protocols and systems to ensure accurate real-time data transmission. Mobile monitoring robot is shown in Fig 6.

Test system hardware including system power test, each module input voltage testing. Positive and negative components before soldering with a multimeter to test the power system short-circuit phenomenon exists, then soldering the power supply part of the circuit, the test two outputs $5 \mathrm{~V}$ voltage is normal, and so on.

Software debugging mainly to the lower part of the control program into the device, and directly connected to the microcontroller serial port to PC, PC to send via serial debugging assistant according to the agreement on the control command to debug the system control section. Whether the controller can properly parse the command and returns the requested data. Control client software were installed into the PC computer before opening the software, the first network connected to the robot's wireless "hot spots", the control terminal and the robot end consisting of a wireless local area network connection parameter settings control terminal for the IP address of the machine the server and port number, if the control terminal after a successful connection, you can preview the real-time video images. Send control commands to test whether the normal motion, video recording and save the picture is normal. The field of video images as shown in Fig 7.

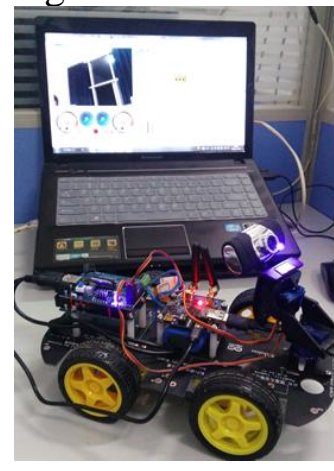

Figure 6: Mobile robot physical map

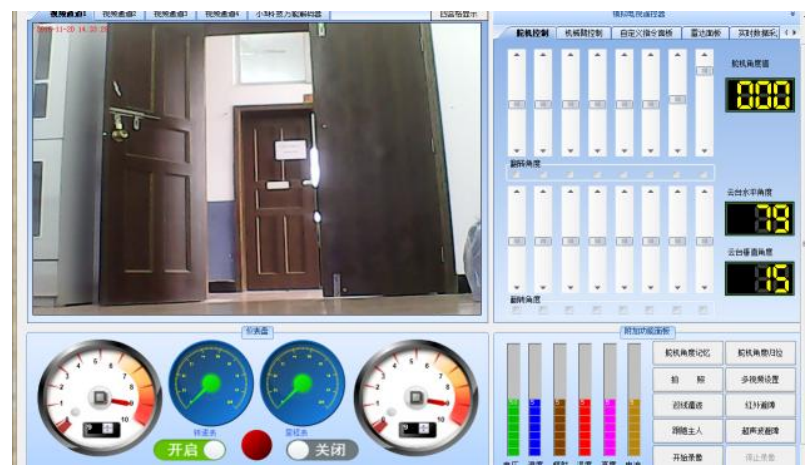

Figure 7: Real time video image 
Based on mechanical design, hardware selection, the mobile robot prototype photographs were developed in the lab. The robot mechanism implemented using four fixed wheels four DC motor control. Arduino UNO R3 microcontroller generates a PWM signal to control the speed and direction of. The robot is given by the command-driven software in the computer system and key algorithms run.

\section{Summary}

This paper is designed and implemented a web-based control of mobile surveillance robots, and describes the implementation of hardware and software. Specific tasks include:

(1) Analysis of the current situation and development trend of robotics technology, combined with the current rapid development of embedded technology and mobile Internet technology, paper proposes a mobile monitoring robot.

(2) Completed the design and commissioning of robot hardware circuit.

(3) The whole system software and hardware test results meet the design goals.

(4) For robot movement in a wireless network environment.

\section{Acknowledgement}

This work was financially supported by the National Natural Science Foundation of China (Grant No. 6154055), the Doctor's scientific research foundation of Hezhou University (No.HZUBS201506).

\section{References}

[1] Kovadic, Z., Cukon, M., Brkic, K., et al, "Design and control of a four-flipper tracked exploration \& inspection robot", Control \& Automation (MED), 2013 21st Mediterranean Conference on , 2013, pp.7-12.

[2] Kasim M. Al-Aubidy, Mohammed M. Ali, Ahmad M. Derbas, et al, "GPRS-Based Remote Sensing and Teleoperation of a Mobile Robot", 10th International Multi-Conference on Systems, Signals and Devices (SSD), Hammamet, Tunisia, 2013,pp.1-7.

[3] Marin, L., Valles, M., Soriano, A., et al, "Event-Based Localization in Ackermann Steering Limited Resource Mobile Robots", Mechatronics, IEEE/ASME Transactions on, vol.19, no.4, 2014, pp.1171-1182.

[4] Han Xiao; Payandeh, S., "Experimental design and analysis in kinematic-based localization in wireless mobile platform network", Systems Conference (SysCon), 2012 IEEE International, 2012, pp.1-6.

[5] Dey, G.K.; Hossen, R.; Noor, M.S. , et al, "Distance controlled rescue and security mobile robot", Informatics, Electronics \& Vision (ICIEV), 2013 International Conference on, 2013, pp.1-6.

[6] Guangming Song, Kaijian Yin, Yaoxin Zhou, et al, "A surveillance robot with hopping capabilities for home security", Consumer Electronics, IEEE Transactions on, vol.55, no.4, 2009, pp.2034-2039.

[7] Sourangsu Banerji, "Design and Implementation of developed an Unmanned Vehicle using a GSM Network with Microcontrollers", International Journal of Science, Engineering and Technology Research (IJSETR) Volume 2, Issue 2, 2013, pp.367-374.

[8] Schmid, K., Hirschmuller, H., "Stereo vision and IMU based real-time ego-motion and depth image computation on a handheld device", Robotics and Automation (ICRA), 2013 IEEE International Conference on, 2013, pp.4671-4678.

[9] Schwartz, F. P., Benac, C., Rocha, V. R. S. , et al, "Microcurrent Stimulation Device Controlled by ATMEGA328P-PU Chip and Android App", 2016 Global Medical Engineering Physics Exchanges/Pan American Health Care Exchanges (GMEPE/PAHCE), 2016.

[10] Derbas, A.M., Al-Aubidy, K.M., Ali. Et al, "Multi-robot system for real-time sensing and monitoring", 15th International Workshop on Research and Education in Mechatronics (REM), El Gouna, Egypt, 2014, pp.1-6. 